MIDPI

sciforum
MOL2NET, International Conference Series on Multidisciplinary Sciences

\section{Deep Learning Applications}

\author{
Cristian R. Munteanu
}

Computer Science Faculty, University of A Coruna, Spain

\section{Abstract.}

The manuscript presents three of my deep learning projects in 2018: mDL-ArtTranfer Deep Learning Art Transfer using Multiple AIs, CNN4Polyps - Colonoscopy polyp detection with Convolutional Neural Networks, Deep-Politics Prediction of Spanish Political Affinity with Deep Neural Nets: Socialist vs People's Party. mDL-ArtTranfer is a mix of adapted scripts using three AI algorithms from fchollet, anishathalye, and ShafeenTejani (GitHub users). Thus, using content images and style pictures, three versions of art transfer will be apply with only one single call.

CNN4Polyps represents the first open GitHub repository for polyp detection and localization into colonoscopy images. The use of small CNNs with 2-3 convolutions, in only 2 minutes with GPU Nvidia Titan Xp, will generate a model with of $92 \%$. The VGG16 transfer learning is no improving this accuracy. The fine-tuning of the last convolutional block and the full-connected layer of the pre-trained Imagenet VGG16 will generate an accuracy over $98 \%$.

Deep-Politics is using the politician's portrait to predict the affinity for two political parties in Spain. Both small CNNs with augmented data and VGG16 transfer learning without data augmentation can generate models with accuracy over $80 \%$. The VGG16 fine-tuning of the last two convolutional blocks and the fullconnected layer will raise the accuracy to $85 \%$. 


\section{Introduction}

Deep Learning (DL) became the standard in image processing [1]. The current applications of DL use the Convolutional Neural Networks (CNN) [2] into three different projects:

- Art transfer: pixel optimization to apply a style to a picture;

- Detection and localization of the colonoscopy polys;

- Prediction of political affinity for two Spanish parties using the politician's portraits.

The results are presented as an open GitHub repositories.

\section{Materials and Methods}

Figure 1 presents the art transfer algorithm using multiple AIs, mDL-ArtTranfer - Deep Learning Art Transfer using Multiple AIs: using a content image, the AI will apply the style from other image, resulting three different version of the initial content image (https://github.com/muntisa/mDLArtTransfer).
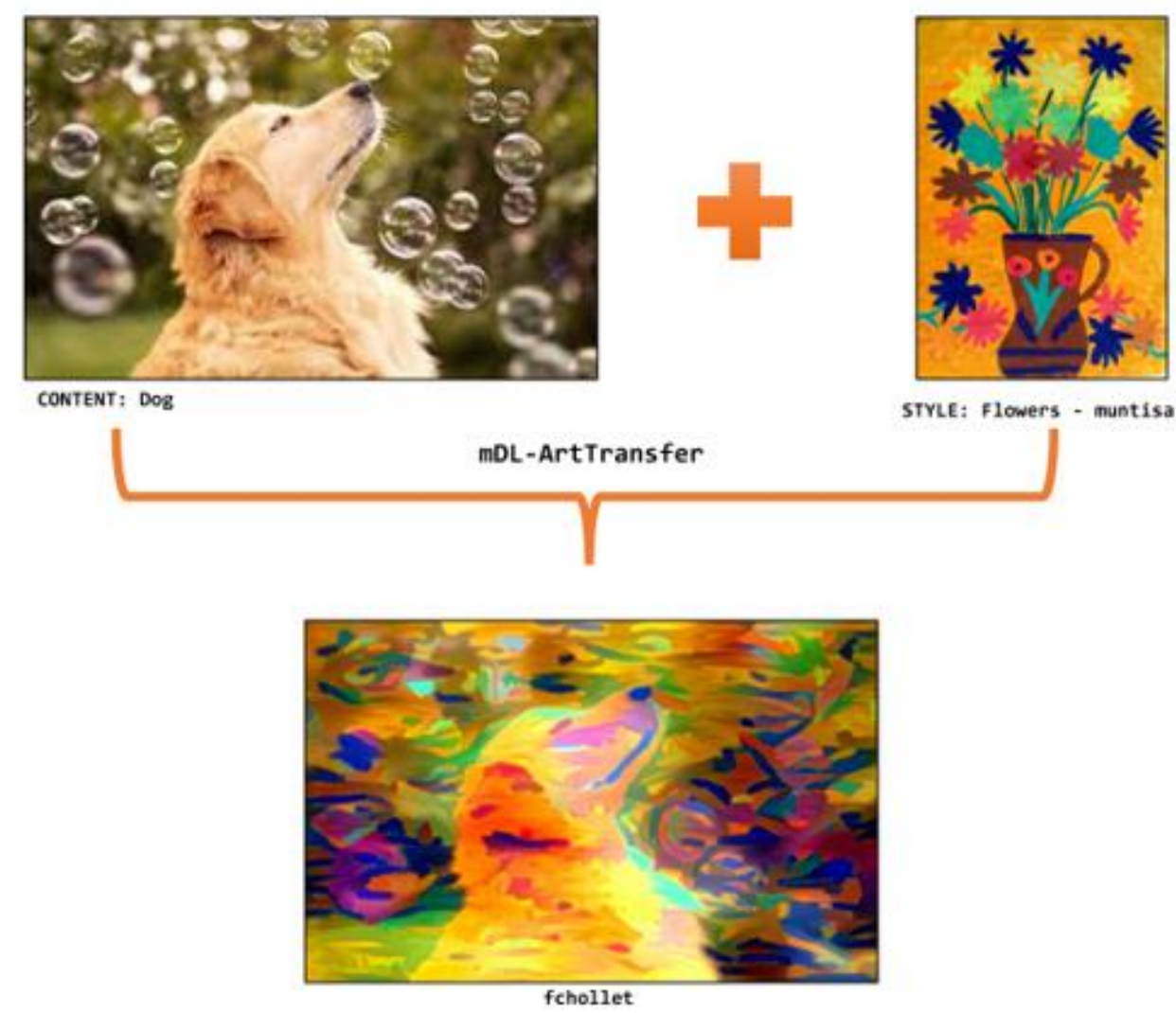

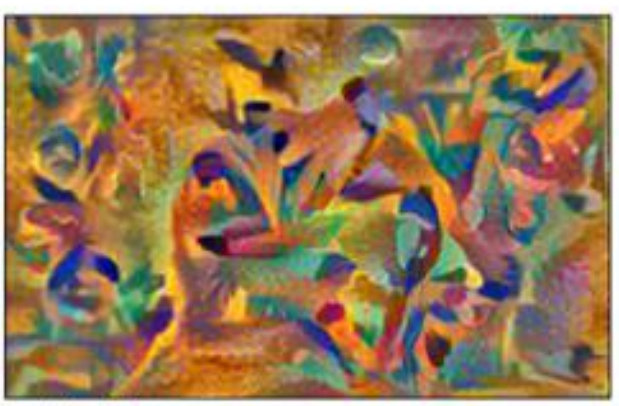

anishathalye

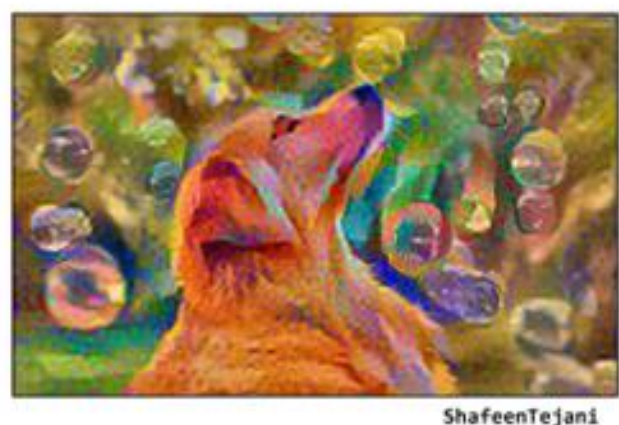

Shafeentejani

Figure 1. mDL-ArtTransfer methodology 
In Figures $\mathbf{2}$ and 3, the flow of the jupyter notebooks are presented, starting with the original colonoscopy images [3] and ending with the polyp localization: CNN4Polyps - Colonoscopy polyp detection with Convolutional Neural Networks (https://github.com/muntisa/Colonoscopy-polypsdetection-with-CNNs).

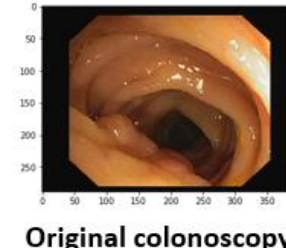

Original colonoscopy (621 images)

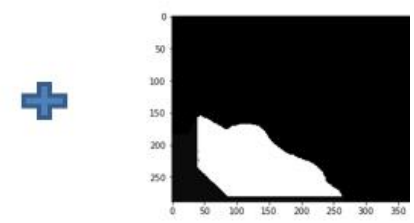

Ground Truth

(621 images)

1-Crop_polyps.ipynb

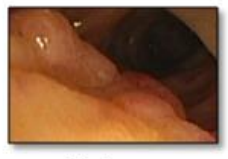

Polyps (606 images)
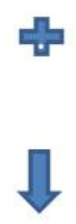

2-Spit_Dataset.ipynb

data polyps

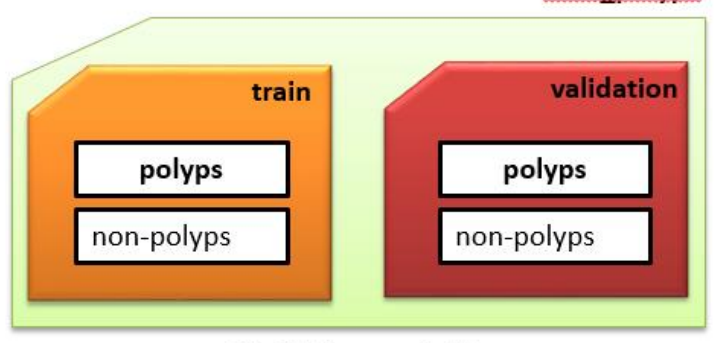

Model dataset folders

(606 images)

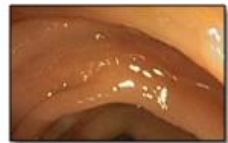

Non-polyps

Figure 2. CNN4Polyps methodology (steps 1-2)
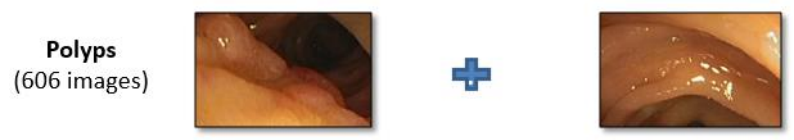

Non-polyps (606 images)

Model dataset folders

3-Sma11_CNNs.ipynb

Small Convolutional Neural Networks (CNNs)

4-TransferLearningVGG16. ipynb

VGG16 Transfer Learning Classifiers

5-FineTuningVGG16.ipynb

VGG16 Fine Tuning Classifiers

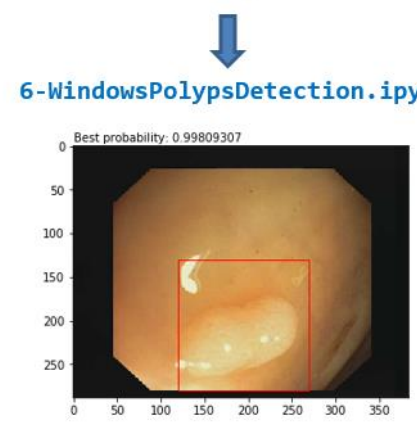

Polyp detection

Figure 3. CNN4Polyps methodology (steps 3-6) 


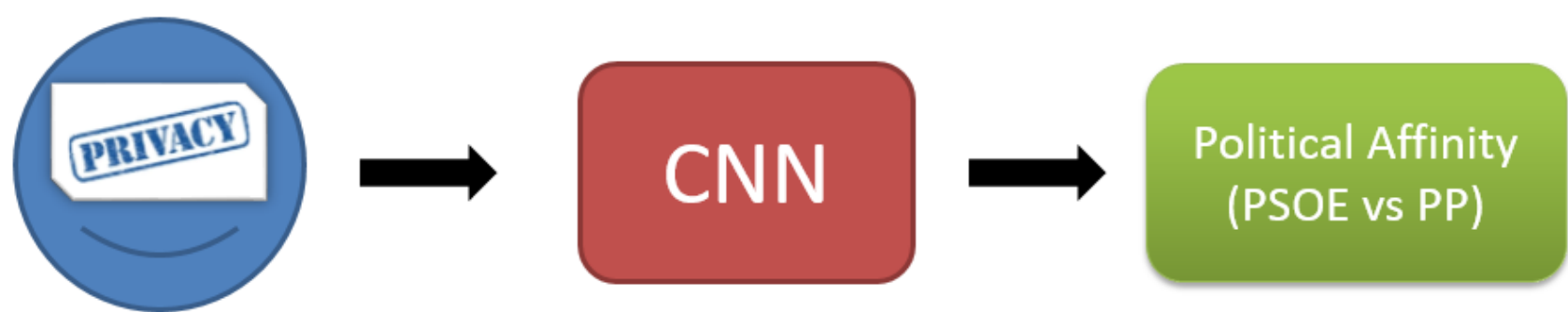

Figure 4. Deep-Politics methodology

Figure 4 represents the CNN methodology using inputs as politician images and output the political affinity: Deep-Politics - Prediction of Spanish Political Affinity with Deep Neural Nets: Socialist vs People's Party (https://github.com/muntisa/Deep-Politics).

\section{Results and Discussion}

Figure 5 presents a sample of the results applying art transfer (mDL-ArtTranfer) to the author portrait:
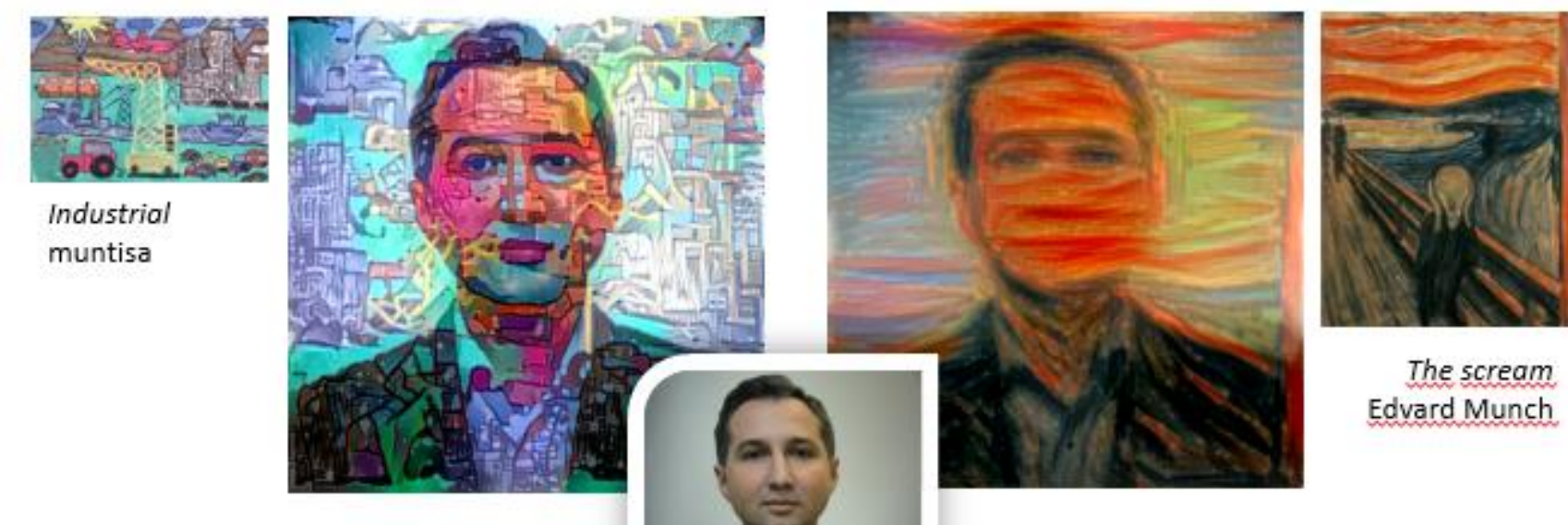

The scream Edvard Munch
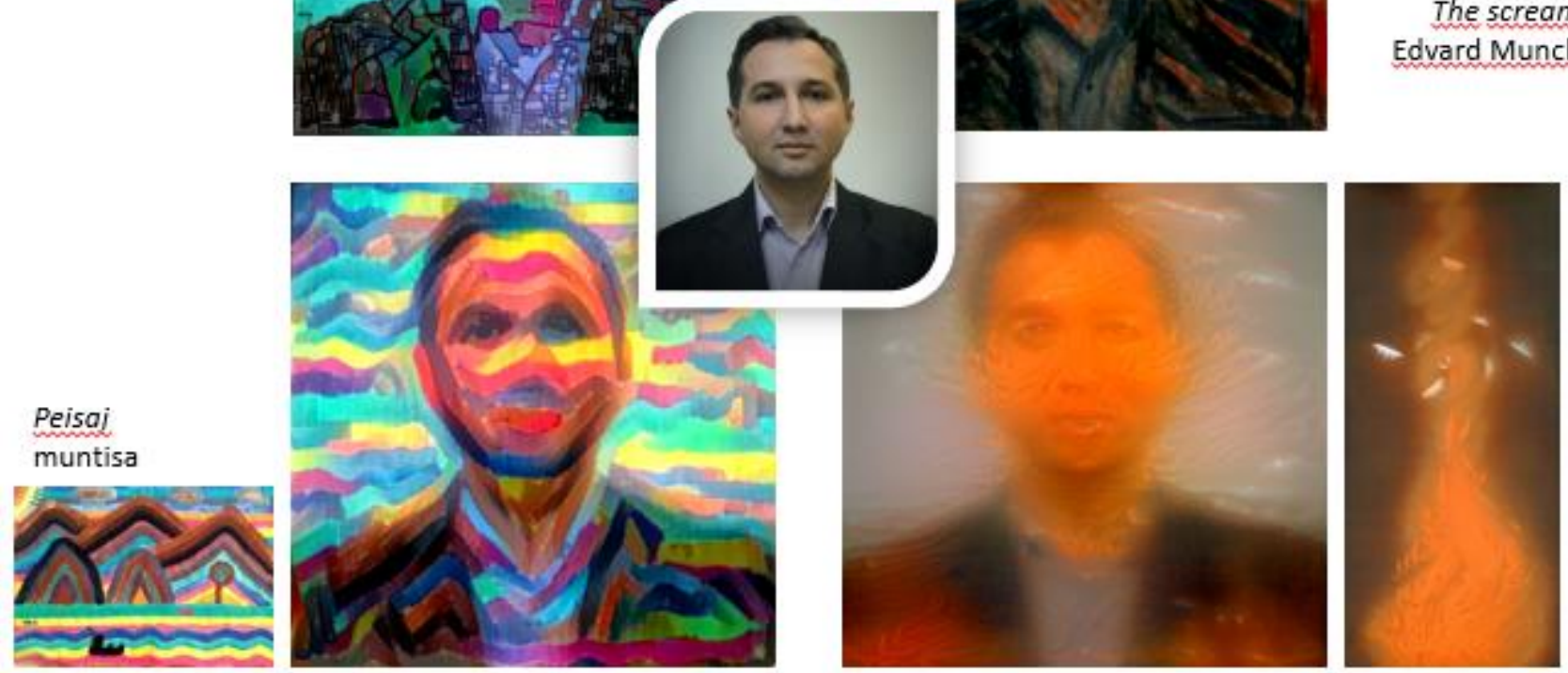

Enbu

Hayami Gyoshu

Figure 5. mDL-ArtTranfer results

An example of colonoscopy polyp detection with CNN4Polyps is presented in Figure 6: the poly is detected with a probability of $99.8 \%$ and localized in the entire colonoscopy image. No predictions are presented with Deep-Politics in order to preserve the personal right of political affinity. 

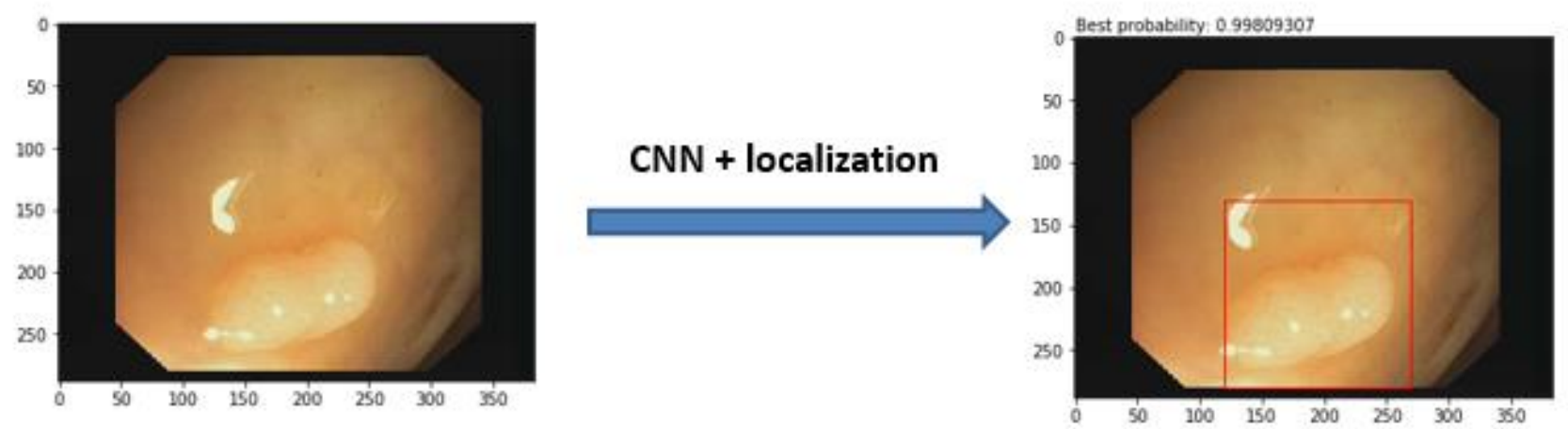

Figure 6. CNN4Polyps poly detection and localization in colonoscopy images

\section{Conclusions}

The presented projects are demonstrating the power of the CNN/DL algorithms, in different fields such as art transfer, medical imaging and politics.

\section{Acknowledgements}

I gratefully acknowledge the support of NVIDIA Corporation with the donation of the Titan Xp GPU used for this research (https://developer.nvidia.com/academic gpu_seeding). The entire project was done at RNASA-IMEDIR, Computer Science Faculty, University of A Coruna, Spain.

\section{References}

[1] Yann LeCun, Yoshua Bengio, Geoffrey Hinton, Deep learning, Nature 521, 436-444 (2015)

[2] Yann LeCun, LeNet-5, convolutional neural networks, http://yann.lecun.com/exdb/lenet/ (2013)

[3] Polyp detection using deep learning, http://site.uit.no/deephealthresearch/2017/07/19/polypdetection-using-deep-learning/ (2017) 